\title{
Design of Intelligent Home Control System Based on GSM Zhang Lizhen ${ }^{1, a,{ }^{*}}$, Xin Haiyan ${ }^{1, b}$, Chen Yulin ${ }^{1, c}$, Huang Haoxia ${ }^{1, d}$ \\ ${ }^{1}$ Southeast University Chengxian College, Nanjing, Jiangsu 210088, China
}

Keyword: GSM; MCU; Intelligent Home

\begin{abstract}
In this paper, the remote monitoring system based on GSM is studied, using AT89S52 microcontroller as the central processing unit, wireless communication using GSM short message. The utility model can realize the function of indoor temperature acquisition, remote lighting control, burglar alarm detector. It provides a solution for the realization of intelligent home.
\end{abstract}

\section{Introduction}

The intelligent home has the unique charm of safe, convenient, efficient, fast, intelligent, individualization. It is of great significance to improve the quality of life of modern people and create a comfortable, safe and convenient living space [1].

With the rapid development of electronic technology, intelligent communication technology and network technology.GSM (Global System for Mobile Communications) because of its unique advantages, such as low cost, strong anti-interference ability, network, received the majority of researchers' attention, provides a shortcut for the realization of the intelligent home control system, has good expansibility and practical value.

This paper studies a kind of home security system based on GSM network and short message platform. It has the functions of residential environment temperature monitoring, home lighting system monitoring and anti-theft system monitoring. Application of this system allows users to control the situation in the home thousands of miles away, both convenient and safe.

\section{System schematic}

The system uses low power consumption, high performance AT89S52 microcontroller as the control core. It makes the corresponding treatment after collecting all kinds of parameters from the peripheral equipments. For example: when the user sends the message to the GSM lighting system through the SIM card, the TC35i module receives the message and sends to the microcontroller in order to process and analyze, and then to control the lights on and off according to the corresponding command[2][3]; When users go out, if someone touches the access control module, the system will send alarm messages to the user, suggesting that a stranger broke into the home; When the user is at home, you can send the appropriate message to close the alarm, to prevent the continuous misleading alarm.

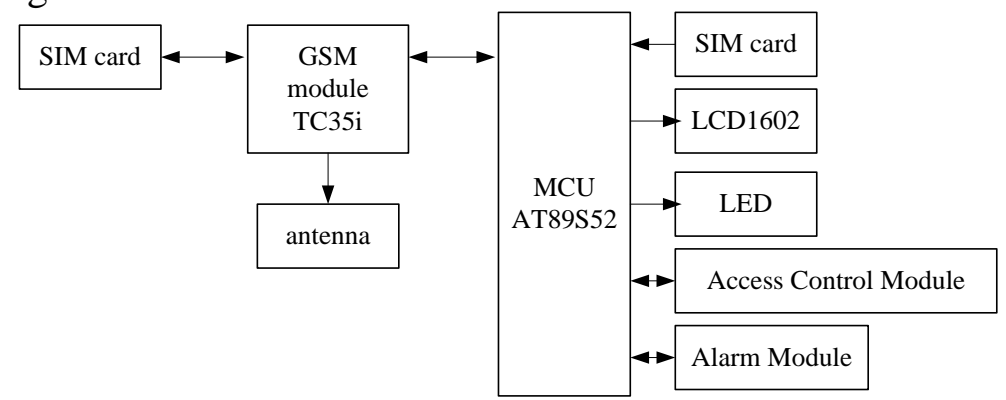

Figure 1 System schematic

The realization of the system is divided into two parts: the hardware part and the software part. The hardware part includes the connection between the controller (AT89S52) and the peripheral 
device, the GSM module and the wireless transceiver module; the software includes the programming of core controller.

\section{Hardware Design}

The hardware of the system is composed of central controller, GSM module and peripheral equipment. The hardware design of the system is as follows:

\subsection{Hardware design of GSM short message module}

The GSM module of the system is the use of industrial GSM module TC35i launched by Siemens, which support Chinese SMS, has its own RS232 communication interface, can easily communicate with the PC machine, single-chip microcomputer, works in the EGSM900 and GSM1800 dual band, can transmit voice and data signals, supports Text and PDU format SMS. Its data interface can transmit commands and data in two-way through the AT command, choosing the baud rate of 300bps 115kbps. The data interface of the system adopts serial asynchronous transceiver, which is in line with the standard of ITU - T RS 232 interface circuit and operates at CMOS level $(2.65 \mathrm{~V})$. The data interface configuration for the 8 bit data bits, 1 bit stop bit, no parity bit, $9600 \mathrm{~b} / \mathrm{s}$, using Text mode to send and receive text messages. Due to the logic level of GSM serial port and the microcontroller is not the same, in practical applications, it is need to add MAX232 level conversion circuit, circuit structure shown in figure 2. The structure of MAX232 level conversion circuit shown in figure 2.

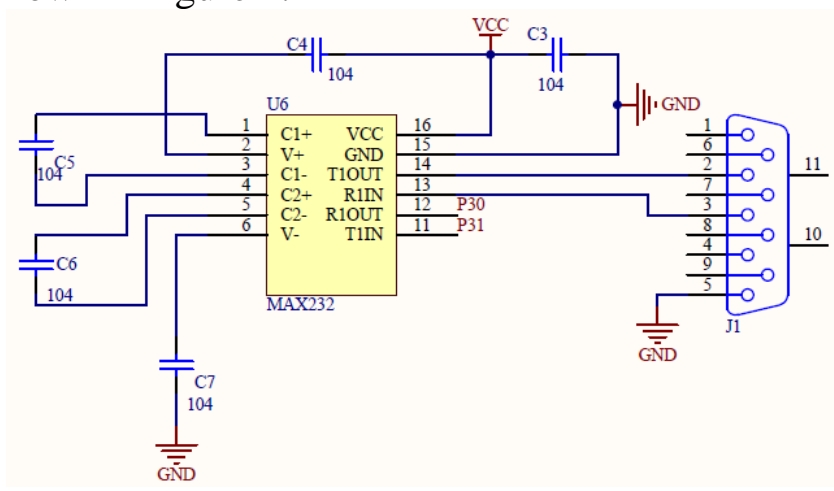

Figure 2 Design of MAX232 level conversion circuit

As shown in the figure, the pin 12 of MAX232 is connected to the P3.0 pin of microcontroller to send data to the microcontroller; The pin 12 of MAX232 is connected to the P3.1 pin of the single chip microcomputer to receive the data sent by the microcontroller; The pin 13 of MAX232 is connected to the serial data output pin of GSM module to receive the data from the GSM module; The pin 14 of MAX232 is connected to the serial data input pin of GSM module to send the data to the GSM module.

\subsection{Hardware design of the lighting system module}

The opening and closing of the lighting system is realized by the closing of the relay, and the circuit of relay control is shown in figure 3. 


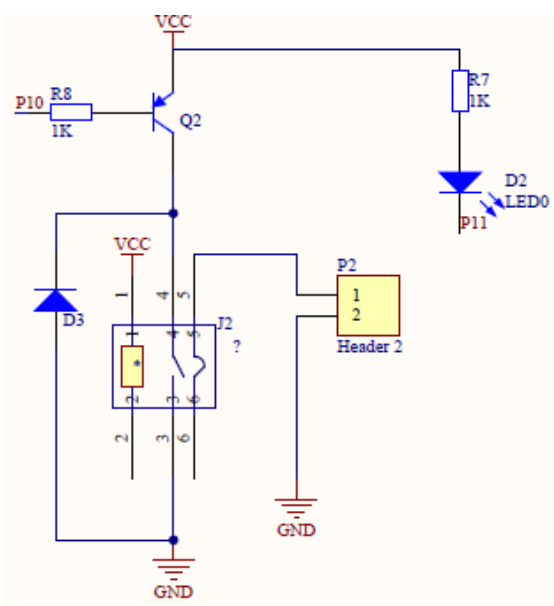

Figure 3 Circuit of relay control

As shown in the figure, P2 is the alarm interface, if the VCC port access to the $5 \mathrm{~V}$ power supply, the transistor conduction. Meanwhile the coil 3-4 is closed, and the relay is driven to pull in, and then 1 and 5 are connected.

\section{Development of system software}

The system software uses the modular design and the $\mathrm{C}$ language programming. The software system mainly includes the software programming of main program module, the lighting control module and the burglar alarm module.

\subsection{Software design of the main module}

The single chip microcomputer is used as the control core of the whole system, carrying the functions of SMS receiving, explanation, transmission and sending control commands to each function module. Among them, the communication of AT89S52 microcontroller and GSM module is the focus of software programming. The communication of AT89S52 microcontroller and GSM module is shown in figure 4.

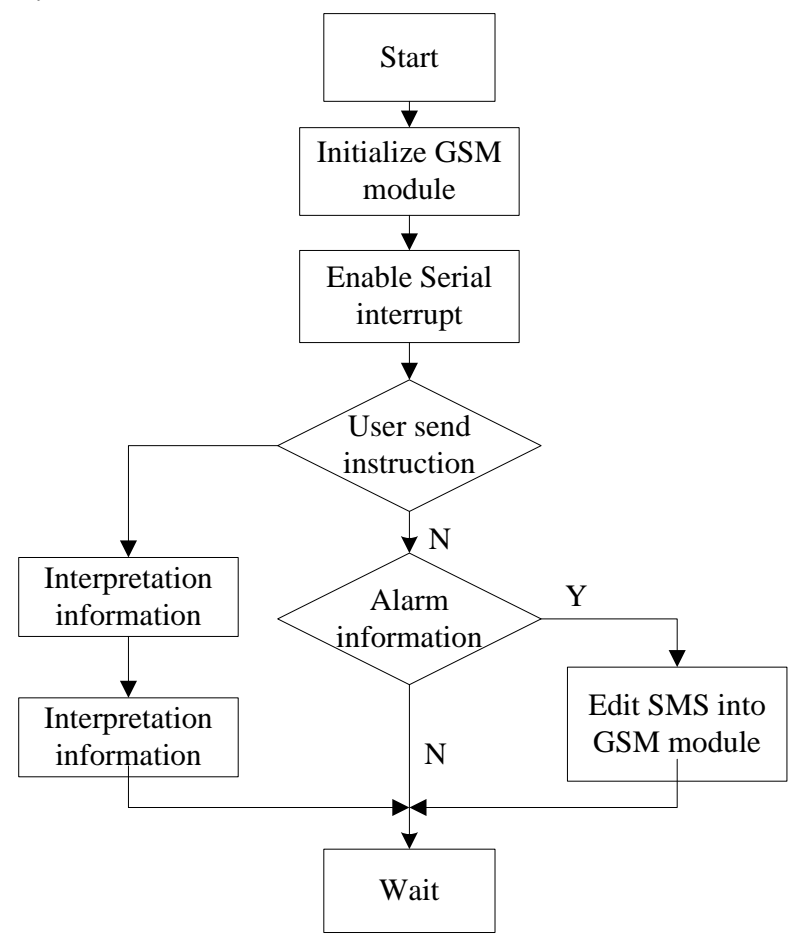

Figure 4 Flow chart of MCU and GSM communication 


\subsection{Software design of lighting module}

The GSM module can receive the short message sent by the mobile phone using TEXT mode, and then extract and analyze the received message content, and real-time processing[4][5]. According to the specific content of the message, such as: SMS for open led, then turn on the lights, SMS for close led, then turn off the lights, so as to control the lights on and off. The specific design flow is shown in figure 5 .

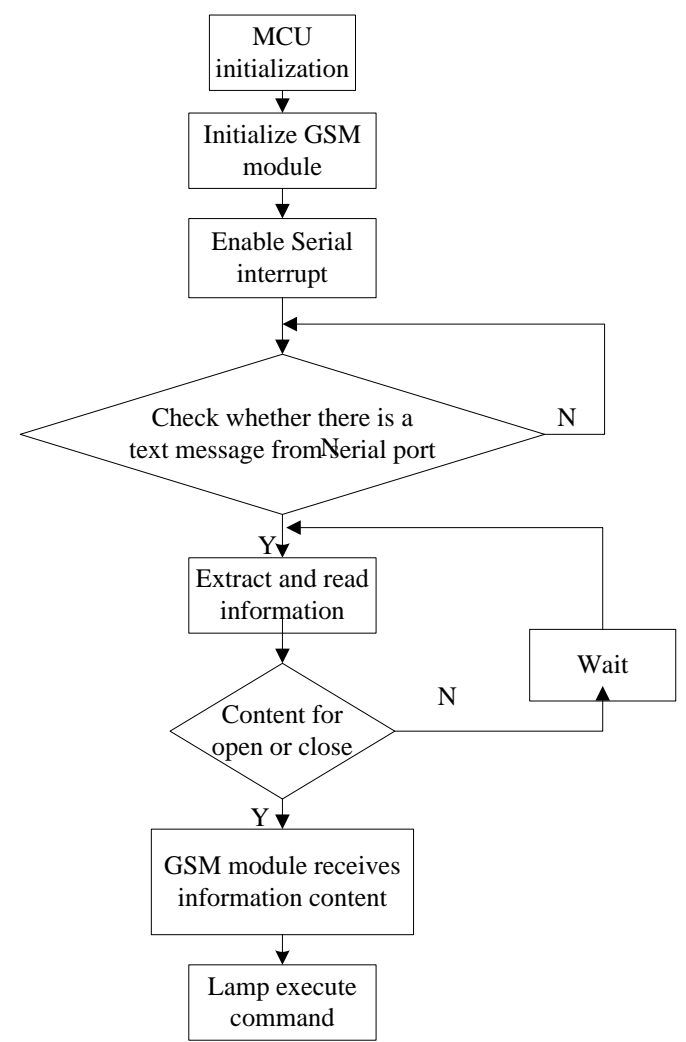

Figure 5 Design flow of Software of lighting module

\subsection{Software design of burglar alarm module}

The user can query the working state of the monitored point by sending short messages, so as to realize the interaction between the person and the monitored object. When the monitored object has an abnormal situation, the alarm controller analyzes the signal to send the alarm short message, and through the GSM module, the mobile phone receives the short message and reads the message. The specific design flow is shown in figure 6. 


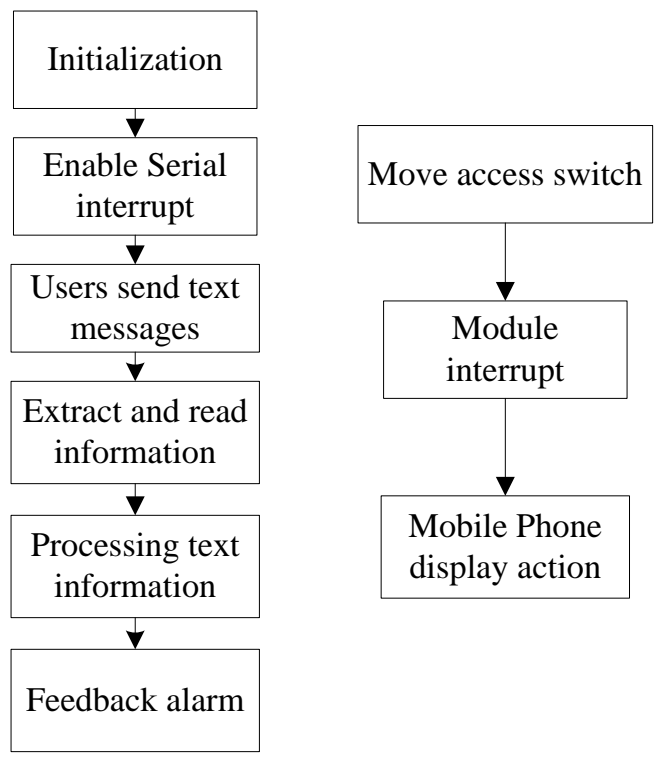

Figure 6 Design flow of burglar alarm module

\section{Conclusions}

This paper introduces a design of intelligent control system based on GSM Home. According to the test results, it can monitor the user's residential environment in real time through SMS, providing a great convenience and guarantee for people's life, and having good expansibility and practical value.

\section{Acknowledgements}

The work described in this paper was supported by General Project of Natural Science Foundation of Colleges and Universities in Jiangsu Province (16KJB510002); Young teachers' Research and Development Foundation of Southeast University Chengxian College (y310001)

\section{References}

[1] Chan M, Campo E, Esteve D, et al. Smart homes-current features and future perspective[J]. Maturitas, 2009,64:900_97

[2] Huang Erlie, Huo Zhenyu, Guo Jia. Smart home controller based on GSM technology [J]. Journal of Liaoning Technical University (NATURAL SCIENCE EDITION), 2008,27 (2).

[3] He Guangyu, Li Taiquan. Design and application of GSM module based on TC35i [J]. Modern Electronic Technology, 2010 (16) (SMS)

[4] Chen Kai, Qin Shihong, Wang Min et al. Design of transceiver control system based on GSM module TC35I [J]. Journal of Wuhan Institute of Technology, 2011,33 (1)

[5] Zhu Hengjun, Zhang Zhihua, Yu Hongbo. Design of intelligent home service system based on GSM SMS early warning [J], Journal of Northeast Petroleum University, 2012,36 (4) 